\title{
The thoughtful self
}

Sheila J. Cunningham

This is the Author's Accepted Manuscript of the book chapter:

Cunninham, S.J. 2018. The thoughtful self. In: G. Hauke \& A. Kritikos (eds.) Embodiment in psychotherapy: a practitioner's guide. Springer.

The final publication is available at Springer via http://dx.doi.org/xxxxxxxxxxxxxxxxxx 


\section{The Thoughtful Self}

\section{Sheila J. Cunningham, Abertay University}

\section{Embodiment definition: The relationship between \\ a concept in the external world (e.g., the self), and its representation in cognition.}

A theoretical understanding of embodiment requires an appreciation of the links between the external environment and the cognitive or thoughtful self, because the embodied self exists at the interface between the outside world and internal cognition. It is therefore important to consider how the self is conceptualised in cognition, and how self processing influences thought. The current chapter aims to provide an overview of the bidirectional relationship between the self and cognition.

The term 'self' is a deceptively simple word. It is used frequently and understood with ease in everyday conversation, and is generally perceived to be basic to our experience as conscious human beings. However, it is a challenging concept to study scientifically - as a psychological construct, the self is deeply intangible, and notoriously difficult to define or measure. Attempts to understand the self from a psychological perspective tend to distinguish between two aspects. First is the subjective self or ' $l$ ', the embodied experience of existing as a continuous entity across time. This can be contrasted with the objective self or 'me', the body of knowledge (e.g., memories, trait characteristics) that are associated with that continuous entity and are considered in the first person. 
Being considerably easier to measure scientifically, the objective self has been the topic of more research than the subjective self. It is possible to measure the emergence and extent of self-knowledge even in pre-verbal humans, and non-human animals. For example, the famous 'mirror recognition test' is argued to show the development of the objective self - a small mark is unobtrusively placed on a child's face, and they are then exposed to their image in a mirror. If the child touches their own face rather than the mirror in response to seeing the unexpected mark, this shows they have understood that the mirror shows their reflection and suggests they can recognise themselves as an object in the world. Later in life, once children are able to verbalise, follow instructions and respond reliably to visual cues, the objective self can be measured more directly by asking about or applying knowledge such as autobiographical memories and character traits.

All kinds of everyday situations trigger the application of self-knowledge. Our awareness of current goals, owned objects and personal tastes guide many actions, and a significant part of our consciousness is devoted to re-living autobiographical events, or planning future activities involving ourselves. If we enter a room and overhear someone saying our name, or perceive another person drinking from our coffee cup, these selfreferent cues have reliable enhancing effects on our attention and memory. These are examples of the biases in cognition associated with self, which ensure that information of relevance to us personally is not missed or forgotten. These biases are the focus of the current chapter. 


\subsubsection{Self-Reference Effects in Attention}

The example above of being able to detect and attend to someone saying your name is an experience most people can relate to. In such a circumstance, it would be extremely difficult to ignore the person using your name, and maintain polite attention to your current conversation. This attention capture by own-names (often referred to as the 'cocktail party effect') is well-established in the laboratory. As early as 1959, Moray described a dichotic listening task in which participants listen to two different passages of information simultaneously, one delivered to each ear. Participants were asked to pay close attention to ether the left or right ear (the 'attended' channel), but when their own name appeared in the 'unattended' channel, they were unable to stop their attention shifting towards it, missing information they were supposed to hear as a result. Many studies have reported similar findings across the years, showing that there is a robust tendency for one's own name to capture attention.

Own-names are perhaps the ultimate self-reference cue, an environmental trigger that alerts us that something is happening which may be of relevance to us. Own-faces also have a similar effect - if you were flicking through a magazine and momentarily caught sight of your own image, you would almost certainly stop flicking and go back to find the glimpsed image. This attention capture is shown by studies in which self-faces are presented alongside images of other faces in search tasks, while brain responses are monitored using electroencephalography (EEG). Participants are significantly faster to find their own face than other images, and when a participant's own face is presented in an experiment, this also evokes a characteristic 'P300' neural signal. The P300 is a change in brain signal that is associated with attention capture - for example, it is evoked by tasks in which an oddball 
stimulus (e.g., a red letter in a series of black letter) appears. This pattern of neural response is therefore consistent with performance on the attention shifting tasks, suggesting that self-images are powerful attention capturing cues.

Speaking to the effectiveness and power of self-referent cues, attention capture can occur even when the self-image is an unwanted distracter. If a participant's own name or face is presented as a distracter while they are attempting to complete a task, this can shift attention away from the current task, even if the participant is trying to consciously avoid changing the focus of their attention. These self-cues are also very effective at sustaining attention - when attention is captured by a self-image, this is not a fleeting response but tends to be maintained across time, perhaps explaining the distracting effect of self-cues.

The pairing of one's own name or face with the self is an association so frequently encountered from a young age that it seems logical that these cues can very quickly and effectively trigger self-referential biases in attention. However, the cues that can trigger selfreferent effects in attention actually extend widely beyond own-names and own-faces. Indeed, even cues that have short-lived and arbitrary associations with self can attract attention as a result of that association. One widely-studied method of assigning selfrelevance to an item is through ownership. Research suggests that when objects are given to participants to own (even temporarily, for the purpose of a game during the experiment) then these objects can evoke self-referent effects on attention. Demonstrating this effect, Turk et al. (2011) presented participants with a shopping game in which images of grocery items were assigned to ownership by the participant or another person. Ownership was indicated by a colour cue that appeared next to the grocery item (e.g., an apple with a blue dot above it would indicate that the 'blue' player owned the apple). Turk et al. measured 
neural signals while participants viewed the images, and found that when the colour cue indicated that the object would be owned by the participant, this evoked the characteristic P300 response that indicates attention capture. Further, there was evidence of additional visual processing of self-owned items, with visuo-spatial attention narrowing to focus on the object and therefore not responding to cues presented elsewhere on-screen. This experiment suggests that there does not have to be a long-held association between self and an external cue for that cue to capture attention; a self-relevant context is sufficient to activate the self-reference effect on attention.

Associated with the attentional effects of self-relevance, other researchers have reported increased perceptual Perceptual processing: The process of observing, processing of items temporarily associated with the self. In one perceiving and interpreting visual stimuli. Occurs with paradigm, Sui, He, and Humphreys (2012) showed that increased speed when visual stimuli are located within the participants are able to identify shapes that represent focus of attention.

themselves more quickly than shapes representing others (imagine you are the triangle, the square is a your friend; Sui et al., 2012). Not only are these self-referenced shapes recognised more quickly, but they are more discernible when they are blurred or distorted that shapes representing other people. As these perceptual effects suggest, the self-reference effect occurs very early in the processing stream. When cues are encountered, they are first perceived by the visual system in the brain, before being identified as self-referent and triggering a reactive response in the brain's attention system. These effects are therefore very quick, automatic and reliable.

The automatic nature of self-reference effects on attention suggests they are a very basic feature of human cognition. This raises the important question of what functional role 
self-processing biases in attention may play. Interestingly, other attention-grabbing stimuli share a common feature of being useful from a survival perspective. For example, threatening images (e.g., angry faces) or goal relevant images (e.g., of food when we are hungry) capture attention for good reasons - in this case, to give us the chance to escape the threat, or meet our goal. It is clearly adaptive to have a system that prioritises attention to these cues. Similarly, it seems logical to suppose that the self-reference effect on attention could be adaptive; we have evolved to live and work in hierarchical social groups and are very sensitive to social information. In such an environment, we need to be very aware of when someone else is discussing information about us, touches items belonging to us, or otherwise acts in a way that has relevance to us. Thus attention-capture by selfrelevant cues serves an adaptive purpose, ensuring that information of potential personal importance is not missed.

The self-referent biases considered above are concerned with early processing responses - fast perception and attention capture. However, in order to be genuinely useful, it is vital that information is successfully retained once it has been attended to. To that end, there are also self-referent biases higher up the cognitive processing stream that ensure self-relevant information is not forgotten once it has been noticed.

\subsubsection{Self-Reference Effects in Memory and Cognition}

When information is encoded in a self-referent context, that information is more likely to be subsequently remembered than similar information encoded in different contexts. This is the classic self reference effect (SRE) in memory, which has been 
established in cognitive psychology since the 1970s. During that decade, many prominent psychologists were following a line of research seeking to clarify what makes information memorable. A general rule to emerge is that the deeper the processing, the greater the memorability. (Deep processing requires meaningful consideration of the incoming information, whereas shallow processing involves little or superficial consideration, as the name implies.) In their seminal depth of processing paper, Craik and Lockhart (1972) showed that the memorability of a list of words could be increased or decreased by changing the processing task at encoding. If participants were asked to consider whether each word had a positive or negative meaning (semantic processing - a 'deep' task) then they were more likely to remember these words than if they had were asked to decide whether they had been presented in lower or upper case letters (a 'shallow' task). These findings established that depth of processing was key to memorability.

Following Craik and Lockhart's proposals, Rogers et al. (1977) proposed the selfreference effect as a depth of processing phenomenon. They contrasted semantic and shallow encoding tasks with a newly-developed self-referential encoding task, in which participants were asked to consider whether or not a trait word was true of themselves (e.g., 'is the word in big letters?' v. 'does the word mean the same as...?' v. 'does the word describe you?'). They found that self-referencing gave rise to better memory than even other deep processing tasks, suggestion that the self provided an exceptional boost to encoding.

In the forty years since Rogers et al. first described the self reference effect on memory, there has been an enormous quantity of research replicating and extending the basic findings. This has confirmed that the self-reference effect is highly robust and reliable. 
Considering character traits in relation to oneself (e.g., "Are you calm?" ) does not just ensure better memory for those traits relative to semantic encoding, but also relative to consideration of other people's characters (e.g., "Is Tom Cruise tidy"?). Interestingly, consideration of very close other referents such as one's mother does moderate the effect; a smaller self-reference effect reliably emerges when the contrasting other person is a close relative than a famous celebrity.

The moderation of the self-reference effect in memory by close others provides some insight into one of the key mechanisms likely to support the effect: the body of person-knowledge stored in long-term memory. Imagine you have been asked whether the word 'modest' describes you. While processing this question, you are likely to Long term memory: The body of information stored in memory for a theoretically unlimited time. Retrieval of information from long term memory can be improved by factors including frequent access, the presence of retrieval cues and storage within an organised network of associated information.

retrieve a rich quantity of autobiographical memories against which you can judge the validity of this characteristic. You may remember specific instances of modest behaviour, or the contrary, and these instances are likely to be relatively vividly recalled. Being asked the same question of your mother or best friend provides the potential to access rich personal memories of shared experiences, but the store of memories will be lower and less accessible than the store of self-referent memories. Being even less familiar, the task of considering whether a famous actor possesses a particular character trait is even less likely to allow access to a rich knowledge base. This means that when you are subsequently asked in a memory test whether you recall the trait words being presented, those you encoded with reference to yourself are 
likely to be associated with the most elaborate memory trace and so are more retrievable than those encoded about the other-referent traits.

The impact of support by the self-knowledge framework was neatly demonstrated by Klein and Loftus (1988), who showed that the self-reference effect is supported by boosts in both elaboration and organisation provided by the self-concept. Klein and Loftus compared the effects of self-referencing with two other strategies, one known to increase memory by encouraging elaboration and one that has the same effect by increasing organisation. The elaboration strategy was implemented by asking participants to learn a list of words (printed on cards) by defining each word in turn. This encourages deep processing, thinking about the details associated with each word in turn to create a rich memory representation. For the organisational strategy, participants were asked to sort the cards into semantic categories. This encourages categorical processing that emphasises links between the words, so when one word is recalled this increases the chances of recalling another from the same category. Finally, a self-referencing strategy was used in which participants considered whether the word reminded them of an autobiographical event. By comparing the pattern of memory performance following the elaboration, organisation and self-referencing for different word sets, Klein and Loftus were able to demonstrate the self-referencing provides both elaboration and organisation support. The body of selfknowledge is so rich and well-connected that it allows us to

Categorical processing: Individual items of information are stored within networks of associated items, creating organised clusters or categories in memory. Activation of a category improves the accessibility of related information because activation spreads through the associative network. Items encoded through categorical processing are therefore more likely to be remembered. 
create rich, elaborate memory representations during encoding, which are organised by their link to the same category (i.e., the self).

Following this early work on the nature of self-reference effects on memory, there was a relatively long period in which the effects were studied largely through the prism of a single paradigm: the trait recall task, requiring conscious consideration of self-knowledge. However, over the past decade there has been a significant increase in the diversity of selfreferencing tasks used to explore memory patterns. In one of the first of these studies, our own lab explored the impact of item ownership on memory (Cunningham, MacDonald, Turk, \& Macrae, 2008). As alluded to above, ownership paradigms are a useful way of exploring self-reference because participants can encode a series of items during a game in which they are temporarily assigned ownership over half of the items. In our study, pairs of participants were asked to imagine they had each won a basket full of shopping (e.g., apples, juice, socks), but they had to sort the items out into a self-owned and other-owned set. This was done on the basis of colour-matching a dot on the card with the colour of each participant's basket. Once all the cards had been sorted, the participants were separated and asked to complete a recognition memory test, in which they were presented with 'selfowned', 'other-owned' and new items. Participants showed a reliable self-reference effect, or 'ownership effect', which we suggested was akin to the classic self-reference effect in memory established by Rogers et al. (1977).

The ownership effect may arise as a result of the same cognitive processes that support self-references trait recall. For example, participants in the ownership paradigm who receive, say, the milk may be accessing their self-knowledge (e.g., "I need to pick up milk at the supermarket on the way home" or "The milk I my cereal was off this morning"). 
However, this seems a much less compelling explanation than in the trait task, as there is no task necessity to reflect on personal knowledge during self-referent trials, and autobiographical memories may also be evoked by the other-referent items ("she got the chocolate biscuits, not me - how annoying!"). Rather, it is likely that attentional prioritising effects may play more of a role here. As described above, when participants perceive that an item belongs to them, this narrows their visuo-spatial attention on the item and elicits a P300 neural response (Turk et al., 2011). This increased attention evoked by self-owned items may be sufficient to boost memory, even without the elaboration and organisation support provided by consideration of self-knowledge in the trait task.

Intrigued by this possibility, a second set of experiments explored what the minimal conditions were in which a self-reference effect on memory could be elicited. This time, we adapted the trait encoding paradigm so that it was presented on screen with either the participant's own face or a celebrity's face, and a trait word positioned above or below the face (Turk, Cunningham, \& Macrae, 2008). In this 'evaluative' version of task, the participant was simply asked whether the trait word described the person shown. This adaptation of Rogers et al.'s paradigm allowed us to exactly match an 'incidental' version of the task, in which participants were simply asked to report whether or not the word was presented above the face. This second version was designed to tests a minimal self-reference effect, because the identity of the referent was completely incidental to the task. Nonetheless, a subsequent recognition memory test showed that the incidental version of the task elicited a self-reference effect on memory: participants were more likely to remember the words shown with their own face, even though they had not been asked to evaluate the trait with reference to the themselves. 
The self-reference effects in memory produced by this range of very different tasks suggests that there may be a range of self-reference effects, supported by different systems. These differences can be drawn out by neuroimaging studies that can distinguish between the cortical activity associated with different SRE tasks, although there are common neural elements. In particular, research across multiple paradigms has identified the medial prefrontal cortex (MPFC) as consistently associated with self-referencing. This area may operate as a 'gateway' to enhanced self-processing. However, different systems are more task-specific - for example, the ownership effect is associated with affect processing and reward networks which are not activated by the trait recall task. It is clear that there is not just one self-processing system, or one way in which the self influences thought. Rather, many different mechanisms (e.g., attention, perception, affective processing, support by self-knowledge) can be heightened when cues of self-relevance are identified, combining to enhance memory.

Given the automaticity of many effects associated with self-processing, it is unsurprising that self-reference effects emerge relatively early in human development. While the trait paradigm precludes the testing of young children (due to language and conceptual difficulties), ownership tasks and other incidental self reference tasks have suggested that children as young as three can show a strong self-bias. We tested this using an adapted ownership task, asking pairs of children to sort out a series of cards into coloured baskets by matching a coloured outline on the card to the colour of the basket. (Cunningham, Vergunst, Macrae, \& Turk, 2013). The participants were each given ownership of one of the baskets (e.g., the red basket) and asked to imagine that the owner of the basket owned all the items (toys, clothes, food) on the card that went into that basket. A 
subsequent recognition memory test showed that participants had a reliable memory advantage for the self-owned items over those owned by the other participant, just like a typical adult sample.

Although self-reference effects on memory have been shown in early childhood, it is not currently clear when these memory effects first emerge. Even very young babies learn to separate themselves and their own body from the external environment (e.g., shown by repeatedly kicking a toy for a rewarding noise), and respond to their own name from about four months. When children begin to vocalise, it is very evident that ownership plays a very important role in early socialisation. The first two-word combinations generally concern personal pronouns and ownership ("my cup", "Mummy's chair") and the majority of disputes between playmates and siblings at two to three years of age concern ownership of desired items ("I had it first!"). Finally, self-descriptions produced by children at this age are reported to include a high number of possessions, such that a three-year-old is more likely to describe himself as 'having blue shoes' when asked who he is than a ten-year-old. The social and personal importance of ownership in the early years serves to highlight how fundamental self-processing systems are in human cognition; their influence may grow across childhood and remain important into old age, but its roots are already measureable at an early stage.

An caveat to the conclusion that self-biases are fundamental and adaptive human systems has emerged recently in the form of cultural differences. Comparisons of selfreference effects in memory from individualistic and collectivist cultures suggests that the reliable self-reference effect widely reported in Western literature is significantly moderated and sometimes even negated in Eastern cultures. Participants in published 
research studies overwhelmingly come from industrialised 'Western' cultures which tend to promote an individualistic culture in which self is considered central (e.g., the USA). Children in these cultures are encouraged to strive to be themselves and follow their dreams, with personal success being a socially acceptable goal. In contrast, collectivist cultures associated with countries such as China and Japan tend to promote achievement as part of a social group, so relations between individuals and their place in the hierarchy are more important than personal characteristics. Participants from collectivist cultures show a reduced selfreference effect, reduced ownership effect, and less self-other differentiation in terms of neural response. There are two potential explanations for this. One is that self-processing biases (i.e., the effects of self on attention and memory) are hard-wired but can be moderated by immersion in a collectivist culture. The second is that self-biases arise as a response to individualistic emphasis in Western culture, so are created rather than naturally emergent. We do not currently have sufficient data to determine which of these explanations is correct, but it is clear that the neural architecture exists for self-reference effects on memory to be robustly exhibited in an individualistic context, even if they can be modulated by culture.

\subsubsection{Our self-directed memories make us who we are (and are not)}

While the effects discussed above concern how our thoughts are influenced by the self, a second central issue is how our thoughts produce our self. There is a strong argument to be made for the perspective that our memories about ourselves create and maintain who we are. The neuroscientist Joseph LeDoux (2003) neatly encapsulates this argument by stating that "[b]ecause you are a unique individual, the particular multifaceted aspects of the 
self that define "you" are present in your brain alone. And in order to remain who you are from minute to minute, day to day, and year to year, your brain must somehow retain the essence of who you are over time. In the end then, the self is essentially a memory, or more accurately, a set of memories." (p. 298). Understanding how the brain deals with selfdirected (i.e., autobiographical) memories is therefore critical to understanding identity.

One of the key issues of any identity system is than of coherence. Individuals tend to have a relatively coherent picture of themselves as possessing personality characteristics (e.g., I am kind but stubborn). This is similar to a stereotype in that it is a structure of person information associated with a Coherence: In the context of self-processing, coherence is the maintenance of a consistent and stable self-concept through the filtering of inconsistent information or memories. certain group (or in this case, individual) which is resistant to change, and alters or filters incoming information to preserve coherence. It is also biased by the rose-tinted spectacles that ensure most people preserve a positive self-concept. For example, when thinking about myself as 'kind', I may bring to mind the time I looked after a friend's child when she was stuck at work, or when I gave my hat to a homeless man in cold weather. However, the times I failed to answer the telephone to that same friend's number, or walked past the homeless man while deliberately looking the other way are not stored with my self-concept because they would challenge this coherent identity. While identity is complex and most people acknowledge that they can display contradictory behaviours at times, a key feature of autobiographical memories is to create the illusion of a coherent personality existing across time. In the inevitable trade-off between accuracy and coherence, the autobiographical memory system seems to err more on the side of coherence. 
The bias toward coherence is encapsulated in Conway's (2005) influential selfmemory system (SMS) model of autobiographical memory. This model describes two elements combining to create and maintain our sense of self. The first system is the store of self-knowledge, here termed the autobiographical knowledge base. This is the repository in long-term memory in which all personal 'episodic' memories are stored, in addition to semantic knowledge derived from autobiographical events (e.g., the episodic memory "I remember my wedding day" can give rise over time to the semantic knowledge "I am married"). Access to this self-relevant material is controlled by the second component of Conway's model, the 'working self', a proposed constituent of working memory. The working self manages a set of active goals, which can increase or decrease the activation of autobiographical knowledge. In line with the coherence argument, the working self is based on the premise that memory is not accurate, what is processed at encoding (and passed to long-term memory) is dependent on congruence with current processing goals. One perpetual current goal is maintenance of the conceptual self, resolving discrepancies between actual behaviour or outcomes, and our ideal selves. In addition, the self balancing the activation of the current goal hierarchy which includes both short term (e.g., "I must post this letter") and long term ("I must get this degree") goals. If an episode is consistent with any of these goals (behaviour that reinforces kind personality; posting of the letter; revising for the exam) then that episode is likely to be stored in long-term memory. If not, like much of daily experience, it is 'destined to be forgotten'. For short term goals, accuracy is more important; for long-term goals, it is coherence that has priority. In Conway's model, the goal hierarchy determines encoding likelihood, the accessibility of knowledge from the autobiographical knowledge base, and the creation of new long-term memories. In other words, according to Conway, this system creates our identity. 
There is evidence that corresponds with the SMS model, such as the fact that people do tend to remember episodes that have either a high degree of correspondence or discord between the events and current goals (e.g., failing an exam or giving birth), and events that either fit with the self-concept or create a self-defining moment (e.g., having a conversation that changes one's career path). However, these patterns are difficult to test empirically as they are associative so causation is difficult to determine - for example, self-defining moments and experiences closely tied to personal goals are likely to be highly emotional, which could also account for their memorability. It seems clear that in line with LeDoux's intuitive explanation, we are what we remember.

Another feature of autobiographical memory that highlights its link to the selfconcept is 'childhood amnesia' or infantile amnesia, the difficulty people have in retrieving memories that were formed before they were three or four years of age. Various explanations for childhood amnesia suggest a role for developing language processing and narrative creation, but an influential and compelling proposal is that childhood amnesia occurs because in the early years, the objective self (with its self-knowledge framework) has yet to develop sufficiently to support the organisation of memories. Before this framework is established, memories can be formed but storage and retrieval quickly disintegrate when there is no organising structure in memory. There are interesting findings consistent with this proposal although, again, empirical evidence is difficult to ascertain because children's development cannot be manipulated. There are associations between the ability to selfrecognise (showing an objective self) and having more reliable memory for events. In one study, Howe, Courage, and Edison (2003) tested young toddlers on two occasions. On the first occasion, the child watched a toy being put in a cupboard. On the second occasion, the 
children's memory for the event was tested by asking them the toy location. It was found that memory was more likely in children who had developed an objective self (again shown by mirror self-recognition). While this evidence remains associative, in combination with the autobiographical memory system described above it is consistent with the bi-directional link between the self and the memory system: what we remember makes us who we are, and who we are directs what we remember.

\subsubsection{The breakdown of the self}

Given the strong, reciprocal links between the self and cognitive processes, it is unsurprising that any severance of the links or damage to one of the components can have a devastating effect on the experience of self. This is perhaps most clearly illustrated by amnesic patients who have lost the ability to form new memories, and who experience a calamitous loss of their sense of self. Sacks (1985) reports a detailed and moving case study of Jimmie G, a 49 year old man who lost the ability to form memories as a result of Korsakoff's syndrome. Jimmie remained effectively stuck in time as a 19 year old, unable to update his memory of himself to form any meaningful understanding of his life or identity as a grown man. In effect, he had lost his self. Sacks describes a traumatic event in which he exposed Jimmie to his own reflection in the mirror. Jimmie was confused and devastated by his mirror image, which showed a man decades older than the self-concept he held in memory. However, he recovered quickly from the distress because he had soon lost all recollection of the mirror event. As Sacks points out, Jimmie's lack of awareness of the loss of himself is telling: "If a man has lost a leg or an eye, he knows he has lost a leg or an eye; 
but if he has lost a self-himself-he cannot know it, because he is no longer there to know $i t^{\prime \prime}$ (p. 34).

An instructive comparison can be made between patients with profound amnesia, and people with dementia disorders such as Alzheimer's disease. As with Sack's amnesic patient Jimmie, patients with dementia can often lose the ability to form new memories and to update their sense of self. Thus cases of mirror misidentification are not unusual, in which the patient is unable to recognise himself or herself in a mirror. As described above, being able to recognise oneself is considered diagnostic of the development of the objective self in childhood, so this seems a particularly profound regression in dementia patients. However, rather than losing their sense of self, there is evidence of some preservation, at least before the late stages of dementia (Pearce, Clare, \& Pistrang, 2002). Patients with Alzheimer's disease, for example, may use first-person speech and recall childhood events despite not being able to remember the previous day. They may also struggle to reconcile current experiences with the stored sense of self, a task that can be made more difficult by the perception of altered social treatment by others. The pattern is closely related to other amnesia experiences, painting a picture of a deteriorating and out-of-date objective self, combined with mismatching preservation of the subjective or embodied self.

As well as self-processing being affected by acquired amnesia or dementia, there are some developmental disorders which may produce atypical patterns of self-reference effects. In particular, autism spectrum disorder (ASD) may change the way in which people process information about both themselves and other people. Children with ASD are able to recognise themselves and can describe autobiographical events, although they may not do this to the same degree as neurotypical children. However, ASD can also affect how the self 
influences cognition, evidenced by difference patterns of self-reference effects in memory than neurotypical controls. For example, people with ASD show reduced a ownership effect in adulthood and some studies suggest a reduced self-reference effect in childhood. ASD is a complex disorder however, encompassing a range of social processing differences, and we have recently found that the strength of self-processing biases depends on the symptoms of the individual (Gillespie-Smith, Ballantyne, Branigan, Turk \& Cunningham, 2018). For participants with mild ASD symptoms, we found a large ownership effect, perhaps as a result of low interest in other people (i.e., not paying much attention to the other person's owned items in the sorting task). In contrast, people with more severe symptoms showed no ownership effect, remembering a similar number of self-owned and other-owned items. This may reflect a more serious disruption of the self-system. While still in their early stages, studies of self-reference effects within populations who show impaired or atypical self- and social-processing patterns can provide much real insight into the complex relationship between self-processing, other-processing and cognition.

\section{Conclusions}

A theoretical understanding of how the self influences thought, and how thought influences cognition, is an important flagstone of the embodies cognition literature. here, we argue that the functional importance of self-cues in the environment have led to their ability to reliably capture and sustain attention, and to the increased memorability of information encoded with reference to self. The self-memory system in particular is critical to our sense of identity, both forming and maintaining our sense of self to the extent that its disruption by neurological damage can be devastated for individuals. By understanding the 
cognitive mechanisms through which the self operates on cognition, we improve our understanding of how the self is created and interacts with the external environment, underpinning models of embodiment.

\section{Clinical Applications:}

The self has an anchoring and directing role in human cognition, so disruption to the selfconcept by clinical conditions can have a profound impact on cognition as well as identity.

One of the most important cognitive links is between the self and memory, so selfprocessing is highly relevant to clinical memory problems. The self-concept provides an organisational structure in long term memory - binding, elaborating and supporting incoming and stored information. It therefore provides some protection against declines in memory linked to disorders like Alzheimer's disease, such that memories associated with the self-concept may remain accessible when other aspects of storing and retrieving information have suffered significant deterioration.

The self is also important as a social construct, so clinical differences in social functioning may be associated with atypical self-processing. In particular, people with autism spectrum disorder may show either an increase or decrease in self-processing bias, depending on their level of social functioning. There are some mixed findings in this area of research but typically, mild autistic traits such as a low interest in or connection with other people increases self-processing biases in memory. However, severe autistic traits may actually reduce the functioning concept of self, leading to the opposite pattern of a reduces self-bias being found. The self concept can be used as a basis for simulating others so disrupted self-processing may be closely related to social processing difficulties more generally.

\section{References}

Conway, M. A. (2005). Memory and the self. Journal of Memory and Language, 53, 594-628.

Craik, F. I. M., \& Lockhart, R. S. (1972). Levels of processing: A framework for memory research. Journal of Verbal Learning and Verbal behavior, 11, 671-684. 
Cunningham, S. J., Turk, D. J., MacDonald, L. M., \& Macrae, C. N. (2008). Yours or mine?

Ownership and memory. Consciousness and Cognition, 17, 312-318.

Cunningham, S. J., Vergunst, F., Macrae, C. N., \& Turk, D. J. (2013). Exploring early selfreferential biases through ownership. British Journal of Developmental Psychology, 31, 289-301.

Howe, M. L., Courage, M. L., \& Edison, S. C. (2003). When autobiographical memory begins. Developmental Review, 23, 471-494.

Gillespie-Smith, K, Ballantyne, C, Branigan, H, Turk, D. J., \& Cunningham, S. J. (2018). The I in Autism: Severity and social functioning in Autism is related to self-processing. British Journal of Developmental Psychology, 36, 127-141.

Klein, S. B., \& Loftus, E. M. (1988). The nature of self-referent encoding: The contribution of elaborative and organizational processes. Journal of Personality and Social Psychology, 55, 5-11.

LeDoux, J. (2003). The self: clues form the brain. Annals of the New York Academy of Sciences, 1001, 295-304.

Moray, N. (1959). Attention in dichotic listening: Affective cues and the influence of instructions. Quarterly Journal of Experimental Psychology, 11, 56-60

Pearce, A., Clare, L., \& Pistrang, N. (2002). Managing sense of self: Coping in the early stages of Alzheimer's disease. Dementia, 1, 173-192.

Rogers, T. B., Kuiper, N. A., \& Kirker, W. S. (1977). Self-reference and the encoding of personal information. Journal of Personality and Social Psychology, 35, 677-688.

Sacks, O. (1985). The man who mistook his wife for a hat. London, Picador. 
Sui, J., He, X., \& Humphreys, G. W. (2012). Perceptual effects of social salience: evidence from self-prioritization effects on perceptual matching. Journal of Experimental Psychology: Human Perception and Performance, 38, 1105-1117.

Turk, D. J., van Bussel, K., Brebner, J. L., Toma, A., Krigolson, O., \& Handy, T. C. (2011). When IT becomes MINE: Attentional biases triggered by object ownership. Journal of Cognitive Neuroscience, 12, 3725-3733. 